\title{
One-step Stochastization Methods for Open Systems
}

\author{
Anna Korolkova ${ }^{1, \star}$ and Dmitry Kulyabov ${ }^{1,2, \star \star}$ \\ ${ }^{1}$ Department of Applied Probability and Informatics, Peoples' Friendship University of Russia \\ (RUDN University), Miklukho-Maklaya 6, 117198 Moscow, Russian Federation \\ ${ }^{2}$ Laboratory of Information Technologies, Joint Institute for Nuclear Research, \\ Joliot-Curie 6, 141980 Dubna, Moscow region, Russia
}

\begin{abstract}
In this paper, two approaches (combinatorial and operatorial) to the stochastization of the one-step processes are discussed for the closed and open version of the Lotka-Volterra model, respectively.
\end{abstract}

\section{Introduction}

Different physical and technical systems can often be modeled as one-step processes [1,2]. Our team has developed a method of stochastization of one-step processes in order to build stochastic models from first principles. For different applications, we used different representations, namely the representation of state vectors (combinatorial approach) [3,4] and the occupation number representation (operatorial approach) [4-14].

In the past we have developed a method for converting ordinary differential equations describing a model into a set of interaction schemes; a technique for obtaining the master equation, the Fokker-Planck equation, the Langevin equation from interaction schemes (combinatorial approach); a diagrammatic technique for deriving the Liouville equation from interaction schemes (operatorial approach). Initially, stochastization methods have been developed for closed Hamiltonian systems. However, when studying open systems, we have found that the developed technique is applicable to open systems as well. In this paper, we demonstrate the applicability of the one-step stochastization method to open systems.

The use of our method is illustrated for the Lotka-Volterra model $[15,16]$. While being extremely simple, this model is a reference mathematical model for a whole class of phenomena $[17,18]$.

\section{The closed system case}

As an example, consider a predator-prey model consisting of animals of two species, one of which hunts down for the other.

In the case of a closed system, the prey is assumed to be provided with inexhaustible food resources $[15,16]$. The deterministic system of equations has the form:

$$
\left\{\begin{array}{l}
\dot{x}=\alpha x-\beta x y, \\
\dot{y}=\beta x y-\gamma y .
\end{array} \quad z=\left(\begin{array}{l}
x \\
y
\end{array}\right) .\right.
$$

\footnotetext{
$\star$ e-mail: korolkova-av@rudn.ru

$\star \star$ e-mail: kulyabov-ds@rudn.ru
} 
Here $x$ is the prey, $y$ is the predator. Preys are reproduced at a rate $\alpha$. When the prey interacts with the predator, the prey die at a rate $\beta$, and the predators reproduced at a rate $\beta$. Predators die at a rate $\gamma$.

The phase trajectory of the system (1) is given in the figure 1 . As we can see, the phase volume is preserved, and non-damping periodic oscillations are established in the system.

We write the interaction scheme for the system (1):

$$
x \stackrel{\alpha}{\rightarrow} 2 x, \quad x+y \stackrel{\beta}{\rightarrow} 2 y, \quad y \stackrel{\gamma}{\rightarrow} 0 .
$$

To find the master equation, we use the combinatorial approach [4].

The master equation for the system (2) is:

$$
\begin{aligned}
\frac{\partial P(x, y, t)}{\partial t}=\alpha(x-1) P(x-1, y, t)+\beta(x+1)(y-1) P(x+1, y-1, t)+ & \\
& +\gamma(y+1) P(x, y+1, t)-(\alpha x+\beta x y+\gamma y) P(x, y, t),
\end{aligned}
$$

where $P(x, y, t)$ is the probability of a system to occupy the state $z=(x, y)$ at the time $t$.

To find the Liouville operator, we may use the operatorial approach [5].

Let $\pi_{x}=1+a_{x}^{\dagger}$ and $a_{x}$ denote the creation and annihilation operators for prey, and $\pi_{y}=1+a_{y}^{\dagger}$ and $a_{y}$ denote the creation and annihilation operators for predators. For interaction schemes, we write diagrams (see figures $3,4,5$ ). The Liouville operator takes the form:

$$
L=\alpha\left(\pi_{x}^{2}-\pi_{x}\right) a_{x}+\beta\left(\pi_{y}^{2}-\pi_{x} a_{y}^{+}\right) a_{x} a_{y}+\gamma\left(1-\pi_{y}\right) a_{y}=\alpha a_{x}^{\dagger}\left(1+a_{x}^{\dagger}\right) a_{x}+\beta\left(a_{y}^{\dagger}-a_{x}^{\dagger}\right)\left(a_{y}^{\dagger}+1\right) a_{x} a_{y}+\gamma a_{y}^{\dagger} a_{y} .
$$

The Liouville equation will have the following form:

$$
\frac{\mathrm{d}|z(t)\rangle}{\mathrm{d} t}=L|z(t)\rangle
$$

where $|z(t)\rangle$ is the state vector at the time $t$.

These are the basic kinetic equation and the Liouville equation for a closed system.

\section{The open system case}

Consider the open predator-prey model. In this case, the food resources for the prey are limited. The deterministic system of equations has the form:

$$
\left\{\begin{array}{l}
\dot{x}=\alpha x-\beta x y-\sigma x^{2}, \quad z=\left(\begin{array}{l}
x \\
y
\end{array}\right) . \\
\dot{y}=\beta x y-\gamma y,
\end{array}\right.
$$

Here, $\sigma$ is the intensity of resource limitation for prey. The phase trajectory of the system (6) is given in figure 2. In this case there is dissipation, in the system there are damped oscillations.

We write down the interaction scheme for the system (6):

$$
x \underset{\sigma}{\stackrel{\alpha}{\rightleftarrows}} 2 x, \quad x+y \stackrel{\beta}{\rightarrow} 2 y, \quad y \stackrel{\gamma}{\rightarrow} 0 .
$$

To find the master equation for (7), we use the combinatorial approach [4] to get

$$
\begin{aligned}
\frac{\partial P(x, y, t)}{\partial t}=\alpha(x-1) P(x-1, y, t)+\beta(x+1)(y-1) P(x+1, y-1, t)+ \\
\quad+\gamma(y+1) P(x, y+1, t)-(\alpha x+\beta x y+\gamma y) P(x, y, t)+\sigma(x+1) x P(x+1, y, t) .
\end{aligned}
$$




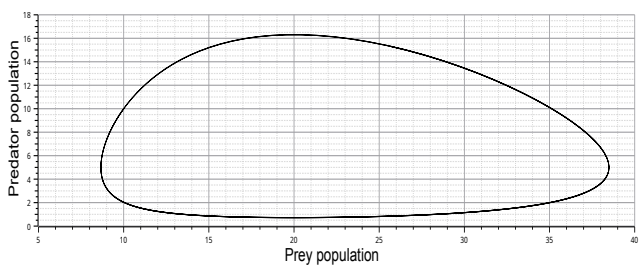

Figure 1. The phase trajectory of the closed system

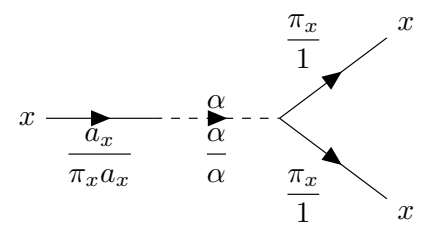

Figure 3. Diagram for interaction $x \stackrel{\alpha}{\rightarrow} 2 x$

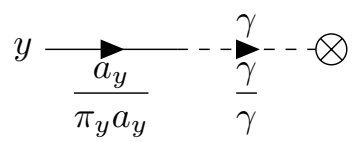

Figure 5. Diagram for interaction $y \stackrel{\gamma}{\rightarrow} 0$

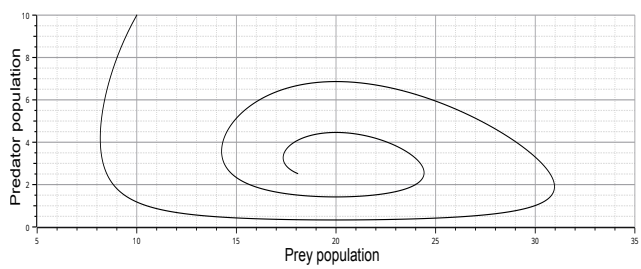

Figure 2. The phase trajectory of the system with dissipation

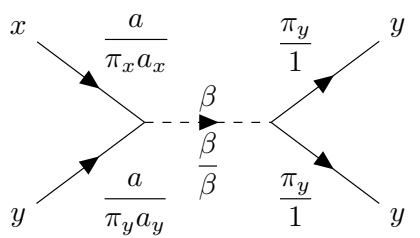

Figure 4. Diagram for interaction $x+y \stackrel{\beta}{\rightarrow} 2 y$

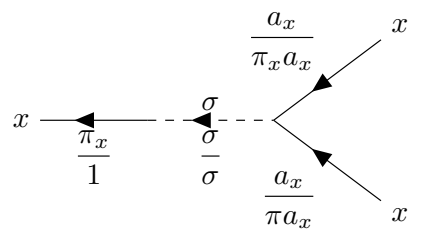

Figure 6. Diagram for interaction $x \stackrel{\sigma}{\leftarrow} 2 x$

To find the Liouville operator, we use the operatorial approach [5]. For an additional interaction scheme, we write the diagram (see figure 6). The Liouville operator takes the form:

$$
\mathcal{L}=\alpha a_{x}^{\dagger}\left(1+a_{x}^{\dagger}\right) a_{x}+\beta\left(a_{y}^{\dagger}-a_{x}^{\dagger}\right)\left(a_{y}^{\dagger}+1\right) a_{x} a_{y}+\gamma a_{y}^{\dagger} a_{y}-\frac{1}{2} \sigma\left(1+a_{x}^{\dagger}\right) a_{x}^{\dagger} a_{x}^{2} .
$$

The Liouville operator (9) for an open system can be written as follows:

$$
\mathcal{L}=L_{0}+L_{D}
$$

where $L_{0}$ is the Liouville operator (4) for the closed system, and $L_{D}$ is the dissipation term.

The Liouville equation has the following form:

$$
\frac{\mathrm{d} \rho(t)}{\mathrm{d} t}=\mathcal{L} \rho(t)
$$

where $\rho(t)$ is the density matrix at the time $t$.

Thus, we wrote down both the basic kinetic equation and the Liouville equation for an open system. 


\section{Conclusion}

The authors previously developed a method of stochastization of one-step processes. Initially, this method was intended for closed Hamiltonian systems only. To obtain the master equation, we used the probabilities of transitions between pure states of the system. To obtain the Liouville equation, a diagrammatic approach was developed using the creation and annihilation operators for the pure states of the system.

As a development of the method, the authors formally applied it to describe open systems. It turned out that the method quite successfully describes open systems as well. To demonstrate, the authors used the universal Lotka-Volterra model. The authors hope that this method will allow solving a wide range of problems in constructing stochastic models.

\section{Acknowledgement}

The publication has been prepared with the support of the "RUDN University Program 5-100".

\section{References}

[1] N.G. van Kampen, Stochastic Processes in Physics and Chemistry, North-Holland Personal Library (Elsevier Science, 2011)

[2] C.W. Gardiner, Handbook of Stochastic Methods: for Physics, Chemistry and the Natural Sciences (Springer Series in Synergetics, 1985)

[3] A.V. Demidova, A.V. Korolkova, D.S. Kulyabov, L.A. Sevastyanov, The Method of Constructing Models of Peer to Peer Protocols, in 6th International Congress on Ultra Modern Telecommunications and Control Systems and Workshops (ICUMT) (IEEE Computer Society, 2015), pp. 557-562, 1504.00576

[4] M. Hnatič, E.G. Eferina, A.V. Korolkova, D.S. Kulyabov, L.A. Sevastyanov, EPJ Web of Conferences 108, 58 (2016), 1603.02205

[5] D.S. Kulyabov, A.V. Korolkova, L.A. Sevastianov, Physics of Atomic Nuclei 81, 916 (2018)

[6] A.V. Korolkova, E.G. Eferina, E.B. Laneev, I.A. Gudkova, L.A. Sevastianov, D.S. Kulyabov, Proceedings 30th European Conference on Modelling and Simulation pp. 698-704 (2016)

[7] P. Grassberger, M. Scheunert, Fortschritte der Physik 28, 547 (1980)

[8] U.C. Täuber, in Ageing and the Glass Transition (Springer Berlin Heidelberg, Berlin, Heidelberg, 2005), Vol. 716, pp. 295-348, 0511743

[9] H.K. Janssen, U.C. Täuber, Annals of Physics 315, 147 (2005), 0409670

[10] M. Mobilia, I.T. Georgiev, U.C. Täuber, Physical Review E 73, 040903 (2006), 0508043

[11] M. Doi, Journal of Physics A: Mathematical and General 9, 1465 (1976)

[12] M. Hnatič, J. Honkonen, T. Lučivjanský, Physics of Particles and Nuclei 44, 316 (2013)

[13] M. Hnatich, J. Honkonen, T. Lučivjanský, Theoretical and Mathematical Physics 169, 1481 (2011)

[14] M. Hnatich, J. Honkonen, T. Lučivjanský, Theoretical and Mathematical Physics 169, 1489 (2011), 1109.6435

[15] A.J. Lotka, Elements of Physical Biology (Williams and Wilkins Company, Baltimore, 1925)

[16] V. Volterra, Leçons sur la Théorie mathématique de la lutte pour la vie (Gauthiers-Villars, Paris, 1931)

[17] W. Feller, Acta Biotheoretica 5, 11 (1939)

[18] F. Schlögl, Zeitschrift für Physik 253, 147 (1972) 